\title{
Analysis of Sound Waves with Semi Perforated Pipe
}

\author{
Burhan Tiryakioglu
}

Faculty of Arts and Sciences, Department of Applied Mathematics, Marmara University, Turkey

\begin{abstract}
The paper presents analytical results of diffraction phenomena at the far field and solution of the wave equation with adequate boundary condition imposed by the pipe wall. An infinite pipe with ring source and perforated part is considered. The solution is obtained by using the Fourier transform technique in conjunction with the Wiener-Hopf method. Applying the Fourier transform technique, the boundary value problem is described by Wiener Hopf equation and then solved analytically.
\end{abstract}

Key words: Wiener-Hopf, Ring Source, Perforated Pipe, Saddle Point

\section{Introduction}

The duct and pipe structures are commonly used in many technical and industrial devices to control the harmful and unwanted noise, such as exhaust systems, ventilation systems, modern aircraft jet and turbofan engines. Hence, the diffraction and radiation of sound waves are very important topic in applied areas and have been subjected to numerous past investigations.

The first study to describe the sound field of an unflanged duct was made by Levine and Schwinger who considered the radiation of sound from rigid cylindrical duct [1]. The reduction of noise in duct systems is generally achieved by silencers. The most well-known of such silencers is acoustically absorbent linings, which have been widely analyzed in literature [2-4]. Another method of reducing noise is to create additional sound absorption by using the perforated structures. Perforated panel or plate, are commonly employed to reduce sound pressure levels across a broad range of applications including industrial installations and propulsion devices [5-7]. In particular, the problem of radiation of sound waves by ducts with perforated structures has been used as a model for many engineering applications, such as noise reduction in exhausts of automobile engines, in modern aircraft jet and turbofan engines, etc. The phenomenon of perforated cylinders has been investigated by various authors, with or without flow. This consideration is important because perforated cylinders provide some facilities for analyzing of sound radiation. Demir and Cinar considered the propagation of sound in an infinite two-part duct carrying mean flow inserted axially into a larger infinite duct with wall impedance discontinuity [8]. In their study, the perforated cylinder properties were investigated and some numerical results were presented.

In the present work a solution is presented for the problem of diffraction of sound waves by an infinite pipe having perforated part. The geometry of the problem under consideration is sketched in Fig. 1. The part $z<0$ of the inner cylinder is hard walled while the part $z>0$ is perforated.

Address: Faculty of Arts and Sciences, Department of Applied Mathematics, Marmara University, 34722, Istanbul, TURKEY. E-mail address: burhan.tiryakioglu@marmara.edu.tr, Phone:+902163451186 
Wiener-Hopf equation is derived using Fourier transform with the help of sophisticated and suitable method known as Wiener-Hopf technique [9].

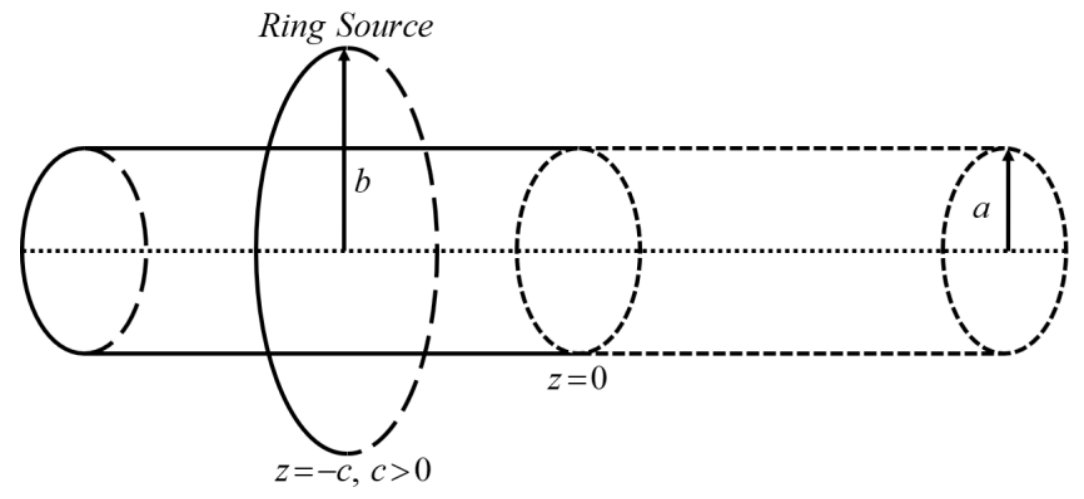

Figure 1. Geometry of the problem

This article is organized as follows. Formulation of the problem and boundary-continuity conditions are described in Section 2. The Wiener-Hopf equation is formed in Section 3 and solved in Section 4. Analysis of the diffracted field is evaluated in Section 5. To end with, conclusions are summarized in Section 6.

\section{Formulation of the Problem}

Consider the diffraction of acoustic waves with an infinite cylindrical pipe system consisting of a two part inner cylinder of radius $r=a$ and a ring source with radius $r=b>a$. Pipe walls are assumed to be infinitely thin and they occupy the region $\{r=a, z \in(-\infty, \infty)\}$ illuminated by a ring source located at $\{r=b>a, z=-c, c>0\}$ (see Fig. 1). The part $z<0$ of the inner cylinder is hard walled while the part $z>0$ is perforated. From the symmetry of the geometry of the problem and of the ring source, the total field will be independent of azimuth $\theta$ everywhere in circular cylindrical coordinate system $(r, \theta, z)$. Therefore, a scalar potential $\psi(r, z)$ which defines the acoustic pressure and velocity by $p=i \omega \rho_{0} \psi$ and $v=\operatorname{grad} \psi$, respectively, is introduced. Here $\rho_{0}$ is the is the density of the undisturbed medium. Time dependence is assumed to be $\exp (-i \omega t)$ and suppressed throughout this paper, where $\omega$ is the angular frequency. For analysis purposes it is convenient to express the total field as

$$
\psi^{T}(r, z)=\left\{\begin{array}{lc}
\psi_{1}(r, z), & r>b \\
\psi_{2}(r, z), & a<r<b \\
\psi_{3}(r, z), & r<a
\end{array}\right.
$$

$\psi_{j}, j=1,2,3$ which satisfy the Helmholtz equation 


$$
\left[\frac{1}{r} \frac{\partial}{\partial r}\left(r \frac{\partial}{\partial r}\right)+\frac{\partial^{2}}{\partial z^{2}}+k^{2}\right] \psi_{j}(r, z)=0, \quad j=1,2,3
$$

is to be determined with the aid of the following boundary and continuity relations. The boundary condition on the rigid surface can be given in terms of the potential functions $\psi_{2}$ and $\psi_{3}$

$$
\begin{aligned}
& \frac{\partial}{\partial r} \psi_{2}(a, z)=0, z<0 \\
& \frac{\partial}{\partial r} \psi_{3}(a, z)=0, z<0
\end{aligned}
$$

Consider now the continuity conditions related to total field at $r=a, z>0$ which are given by

$$
\begin{gathered}
\frac{\partial}{\partial r} \psi_{2}(a, z)-\frac{\partial}{\partial r} \psi_{3}(a, z)=0, z>0 \\
\psi_{2}(a, z)-\psi_{3}(a, z)=i \frac{\zeta_{p}}{k} \frac{\partial}{\partial r} \psi_{3}(a, z), z>0
\end{gathered}
$$

where $\zeta_{p}$ is the specific impedance, describing the acoustic properties of the perforated screen. For stationary media, the empirical formula of the specific acoustic impedance $\zeta_{p}$ is given by [5]

$$
\zeta_{p}=\left[0.006-i k\left(t_{w}+0.75 d_{h}\right)\right] / \sigma
$$

where $t_{w}$ is the screen thickness, $d_{h}$, the perforate hole diameter and $\sigma$ the porosity. By the definition of the ring source given as

$$
\begin{aligned}
\frac{\partial}{\partial r} \psi_{1}(b, z)-\frac{\partial}{\partial r} \psi_{2}(b, z) & =\delta(z+c),-\infty<z<\infty \\
\psi_{1}(b, z)-\psi_{2}(b, z) & =0,-\infty<z<\infty
\end{aligned}
$$

where $\delta$ is dirac delta function.

\section{Derivation of the Wiener-Hopf Equation}

The unknown fields $\psi_{1}(r, z), \psi_{2}(r, z)$ and $\psi_{3}(r, z)$ satisfy (2) for $z \in(-\infty, \infty)$. By taking Fourier transform of these equations, one can obtain the following integral representations

$$
\psi_{1}(r, z)=\frac{k}{2 \pi} \int_{L} A(\alpha) H_{0}^{(1)}(\lambda k r) \exp (-i \alpha k z) d \alpha
$$




$$
\begin{gathered}
\psi_{2}(r, z)=\frac{k}{2 \pi} \int_{L}\left[B(\alpha) J_{0}(\lambda k r)+C(\alpha) Y_{0}(\lambda k r)\right] \exp (-i \alpha k z) d \alpha \\
\psi_{3}(r, z)=\frac{k}{2 \pi} \int_{L} D(\alpha) J_{0}(\lambda k r) \exp (-i \alpha k z) d \alpha
\end{gathered}
$$

where $L$ is a suitable inverse Fourier transform integration contour along or near the real axis in the complex $\alpha$-plane. $A(\alpha), B(\alpha), C(\alpha)$ and $D(\alpha)$ are spectral coefficients to be determined. $J_{0}$ and $Y_{0}$ are the Bessel and Neumann functions of order zero and $H_{0}^{(1)}=J_{0}+i Y_{0}$ is the Hankel function of the first type. $\lambda$ is square root function which is defined as

$$
\lambda(\alpha)=\sqrt{1-\alpha^{2}}, \operatorname{Im}(\lambda) \geq 0
$$

Branch cuts for $\lambda$ is taken on the line from 1 to $\infty$ and from $-\infty$ to -1 . As usual in this kind of Wiener-Hopf problem, we will assume that the surrounding medium is slightly lossy and $k$ has a small positive imaginary part. The lossless case can be obtained by letting $\operatorname{Im} k \rightarrow 0$ at the end of the analysis. Applying the boundary condition (4) on $r=a$ and taking Fourier transforms gives

$$
-D(\alpha) \lambda k J_{1}(\lambda k a)=\Phi^{+}(\alpha)
$$

Continuity relation at $r=a$ yields

$$
\begin{gathered}
{[D(\alpha)-B(\alpha)] J_{1}(\lambda k a)=C(\alpha) Y_{1}(\lambda k a)} \\
{[D(\alpha)-B(\alpha)] J_{0}(\lambda k a)-C(\alpha) Y_{0}(\lambda k a)=\Phi^{-}(\alpha)-i \frac{\zeta_{p}}{k} \Phi^{+}(\alpha)}
\end{gathered}
$$

where $\Phi^{+}$and $\Phi^{-}$are a function analytic at the upper $(\operatorname{Im} \alpha>0$ or $\operatorname{Im} \alpha=0$ and $\operatorname{Re} \alpha>0)$ and lower $(\operatorname{Im} \alpha<0$ or $\operatorname{Im} \alpha=0$ and $\operatorname{Re} \alpha<0)$ half plane and defined as

$$
\begin{gathered}
\Phi^{+}(\alpha)=\int_{0}^{\infty} \frac{\partial}{\partial r} \psi_{3}(a, z) \exp (i \alpha k z) d z \\
\Phi^{-}(\alpha)=\int_{-\infty}^{0}\left[\psi_{2}(a, z)-\psi_{3}(a, z)\right] \exp (i \alpha k z) d z
\end{gathered}
$$

The spectral coefficient $D(\alpha)$ can be found easily from (14) while $A(\alpha), B(\alpha)$ and $C(\alpha)$ are related to each other by the definition of the ring source given in $(8,9)$, application of the boundary conditions on $r=b$ yields

$$
\begin{gathered}
\lambda k A(\alpha) H_{1}^{(1)}(\lambda k b)=\lambda k B(\alpha) J_{1}(\lambda k b)+\lambda k C(\alpha) Y_{1}(\lambda k b)-\exp (-i \alpha k c) \\
A(\alpha) H_{0}^{(1)}(\lambda k b)=B(\alpha) J_{0}(\lambda k b)+C(\alpha) Y_{0}(\lambda k b)
\end{gathered}
$$


From the relations (19) and (20), we obtain

$$
\begin{aligned}
& B(\alpha)=A(\alpha)+\exp (-i \alpha k c) \frac{\pi b}{2} Y_{0}(\lambda k b) \\
& C(\alpha)=i A(\alpha)-\exp (-i \alpha k c) \frac{\pi b}{2} J_{0}(\lambda k b)
\end{aligned}
$$

(14) and (15) allow us to express the unknown coefficient $A(\alpha)$ in terms of the analytic function $\Phi^{+}(\alpha)$ as follows

$$
A(\alpha)=-\frac{\Phi^{+}(\alpha)}{\lambda k H_{1}^{(1)}(\lambda k a)}+\frac{\pi b \exp (-i \alpha k c)}{2 H_{1}^{(1)}(\lambda k a)}\left[J_{0}(\lambda k b) Y_{1}(\lambda k a)-Y_{0}(\lambda k b) J_{1}(\lambda k a)\right]
$$

The substitution of $B(\alpha), C(\alpha)$ and $D(\alpha)$ into (16) yields

$$
\begin{aligned}
A(\alpha) H_{0}^{(1)}(\lambda k a)= & \Phi^{+}(\alpha)\left[i \frac{\zeta_{p}}{k}-\frac{J_{0}(\lambda k a)}{\lambda k J_{1}(\lambda k a)}\right] \\
& -\Phi^{-}(\alpha)+\exp (-i \alpha k c) \frac{\pi b}{2}\left[J_{0}(\lambda k b) Y_{0}(\lambda k a)-Y_{0}(\lambda k b) J_{0}(\lambda k a)\right]
\end{aligned}
$$

Inserting now (23) into (24) we get the following Wiener-Hopf equation:

$$
\Phi^{+}(\alpha) M(\alpha)+\Phi^{-}(\alpha)=\exp (-i \alpha k c) \frac{b}{a} \frac{H_{0}^{(1)}(\lambda k b)}{\lambda k H_{1}^{(1)}(\lambda k a)}
$$

where

$$
M(\alpha)=\frac{J_{0}(\lambda k a)}{\lambda k J_{1}(\lambda k a)}-\frac{H_{0}^{(1)}(\lambda k a)}{\lambda k H_{1}^{(1)}(\lambda k a)}-i \frac{\zeta_{p}}{k}
$$

\section{Solution of the Wiener-Hopf Equation}

Consider the Wiener-Hopf equation in (25) and rearrange it using (26) in the following form

$$
\Phi^{+}(\alpha) M^{+}(\alpha)+\Phi^{-}(\alpha) M^{-}(\alpha)=\exp (-i \alpha k c) \frac{b}{a} M^{-}(\alpha) \frac{H_{0}^{(1)}(\lambda k b)}{\lambda k H_{1}^{(1)}(\lambda k a)}
$$

Here, $M^{+}(\alpha)$ and $M^{-}(\alpha)$ are the split functions regular and free of zeros in the upper and lower half planes, respectively, resulting from the Wiener-Hopf factorization of $M(\alpha)$ as [10] 


$$
M(\alpha)=M^{+}(\alpha) / M^{-}(\alpha)
$$

Now consider (27), by using the classical decomposition procedure for complex term, one gets

$$
\Phi^{+}(\alpha) M^{+}(\alpha)+\Phi^{-}(\alpha) M^{-}(\alpha)=Q^{+}(\alpha)+Q^{-}(\alpha)
$$

Decomposing $Q(\alpha)$ we obtain split functions $Q^{+}(\alpha)$ and $Q^{-}(\alpha)$ which are regular in the upper and lower half planes, respectively.

$$
Q(\alpha)=\exp (-i \alpha k c) \frac{b}{a} M^{-}(\alpha) \frac{H_{0}^{(1)}(\lambda k b)}{\lambda k H_{1}^{(1)}(\lambda k a)}=Q^{+}(\alpha)+Q^{-}(\alpha)
$$

The Wiener-Hopf equation in (29), yields

$$
\Phi^{+}(\alpha) M^{+}(\alpha)-Q^{+}(\alpha)=-\Phi^{-}(\alpha) M^{-}(\alpha)+Q^{-}(\alpha)
$$

Now both sides of (31) are analytical functions on upper and lower regions. From analytical continuation principle and Liouville's theorem, we get the Wiener-Hopf solution

$$
\Phi^{+}(\alpha)=Q^{+}(\alpha) / M^{+}(\alpha)
$$

\section{Analysis of the Diffracted Field}

The total field in the region $r>b$ can be obtained from (10)

$$
\psi_{1}(r, z)=\frac{k}{2 \pi} \int_{L} A(\alpha) H_{0}^{(1)}(\lambda k r) \exp (-i \alpha k z) d \alpha
$$

Inserting $A(\alpha)$ into (33) and utilizing the asymptotic expansion of $H_{0}^{(1)}(\lambda k r)$ as $k r \rightarrow \infty$

$$
H_{0}^{(1)}(\lambda k r)=\sqrt{\frac{2}{\pi \lambda k r}} \exp (i \lambda k r-i \pi / 4)
$$

and using the saddle point technique, one obtains

$$
\begin{aligned}
\psi_{1}(R, \theta) & =\frac{i}{\pi} \frac{\Phi^{+}\left(-\cos \theta_{1}\right)}{\sin \theta_{1} H_{1}^{(1)}\left(k a \sin \theta_{1}\right)} \frac{\exp \left(i k R_{1}\right)}{k R_{1}} \\
& -\frac{i k b}{2} \exp \left(i k c \cos \theta_{1}\right) \frac{\left[J_{0}\left(k b \sin \theta_{2}\right) Y_{1}\left(k a \operatorname{in} \theta_{2}\right)-Y_{0}\left(k b \operatorname{in} \theta_{2}\right) J_{1}\left(k a \text { in } \theta_{2}\right)\right]}{H_{1}^{(1)}\left(k a \sin \theta_{2}\right)} \frac{\exp \left(i k R_{1}\right)}{k R_{1}}
\end{aligned}
$$


where $\Phi^{+}(\alpha)$ is given by (32). $R_{1}, R_{2}$ and $\theta_{1}, \theta_{2}$ are the spherical coordinates defined by

$$
r=R_{1} \sin \theta_{1}, z=R_{1} \cos \theta_{1}
$$

and

$$
r=R_{2} \sin \theta_{2}, z+c=R_{2} \cos \theta_{2}
$$

\section{Conclusions}

The diffraction of sound waves emanating from a ring source by a semi-infinite pipe, whose parts $z<0$ and $z>0$ are hard walled and perforated, respectively is investigated rigorously by the Wiener-Hopf method. A semi perforated pipe was considered. The problem is modelled two dimensional due to symmetry of the geometry. The problem is formulated into a Wiener-Hopf equation. This equation is solved analytically with the help of Wiener-Hopf technique.

In forthcoming study, to a better understanding the effect of perforated part on the diffracted field, numerical calculations and graphics are going to be obtained.

\section{References}

[1] Levine H, Schwinger J. On the radiation of sound from an unflanged circular pipe. Physical Review 1948;73:383-406.

[2] Rawlins AD. Radiation of sound from an unflanged rigid cylindrical duct with an acoustically absorbing internal surface. Proc. Roy. Soc. Lond. A. 1978;361:65-91.

[3] Tiryakioglu B, Demir A. Radiation analysis of sound waves from semi-infinite coated pipe. International Journal of Aeroacoustics 2019;18:92-111.

[4] Tiryakioglu B, Demir A. Sound wave radiation from partially lined duct. Archives of Acoustics 2019;44:239-249.

[5] Sullivan JV, Crocker MJ. Analysis of concentric-tube resonators having unpartitioned cavities. Journal of the Acoustical Society of America 1978;64:207-215.

[6] Yang C, Cheng L, Hu Z. Reducing interior noise in a cylinder using micro-perforated panels. Applied Acoustics 2015;95:50-56.

[7] Tiryakioglu B. Sound radiation from the perforated end of a lined duct. Acta Acustica united with Acustica 2019;105:591-599.

[8] Demir A, Cinar OY. Propagation of sound in an infinite two-part duct carrying mean flow inserted axially into a larger infinite duct with wall impedance discontinuity. ZAMM - Journal of Applied Mathematics and Mechanics 2009;89:454-465.

[9] Noble B. Methods Based on the Wiener-Hopf Technique. Pergamon Press, London; 1958.

[10] Demir A, Rienstra SW. Sound radiation from a lined exhaust duct with lined afterbody. AIAA-2010-3947 of the 16th AIAA/CEAS Aeroacoustics Conference, Stockholm, Sweden, 79 June 2010. 